\section{Some News on Old Concepts and the Epigenetics of Alzheimer's Disease}

Received: December 14, 2017; Accepted: December 15, 2017; Published: December 18,2017

\section{Editorial}

Alzheimer's disease in the most important neurodegenerative disorder worldwide and a major problem of health in developed countries, representing the $6^{\text {th }}$ cause of death in the USA, with an age-adjusted death rate of 25.4 per 100,000 . Its prevalence progresses with age, ranging from $1.8 \%$ at $65-69$ years to $42.1 \%$ at age 95-99 years, with an annual incidence of 34.1 per 1,000 persons $>60$ years) [1-3]. Total costs of AD rise from $€ 9,000$ at 6 months to over $€ 21,000$ per patient 2 years later [4]. Approximately $10-20 \%$ of the total cost of dementia is due to pharmacological treatment; however, current drugs are not costeffective and no new drugs for $A D$ have been approved by the FDA during the past 15 years [5]. The pharmacological treatment of $A D$ has been dominated by cholinesterase inhibitors (Tacrine, Donepezil, Rivastigmine, Galantamine) since the introduction of Tacrine in the market in 1993, based on the assumption that $A D$ was a cholinergic deficiency caused by selective neurodegeneration of the nucleus basalis of Meynert. In the early 2000's, Memantine, a non-competitive NMDA receptor antagonist, was approved for the treatment of severe dementia. Since the identification of the disease by Alois Alzheimer in 1906, the confronted, dominant pathogenic theories of $A D$ were the amyloidopathy and tauopathy responsible for conformational changes in the amyloid-beta $(A \beta)$ protein and the hyperphosphorylation of the tau protein, respectively, leading to the phenotypic expression of extracellular amyloid deposits in senile plaques and the intracellular formation of neurofibrillary tangles (NFTs), as the major pathogenic hallmarks of AD [6]. The amyloid hypothesis was reinforced by the identification in 1987 of point mutations in the Amyloid Precursor Protein (APP) gene whose abnormal processing by $\alpha-, \beta-$, and $\gamma$-secretases and posttranscriptional changes gives rise to the $A \beta$ deposits [7]. Years later, other pathogenic genes were identified, such as those encoding presenilin-1 (PSEN1) and presenilin-2 (PSEN2); and at present, more than 600 different genes distributed across the human genome are believed to be associated with premature neuronal death and neurodegeneration in $A D$ [8-10]. Among these, in the early 1990's, the late Allen Roses proposed the gene encoding apolipoprotein E (APOE) as the most important risk factor in those patients harboring the APOE-4 allele, which is involved in different pathogenic events associated with neurodegeneration and vascular dysfunction [10]. In parallel
Ramón Cacabelos ${ }^{1,2 *}$

1 Institute of Medical Science and Genomic Medicine, EuroEspes Biomedical Research Center, Corunna, Spain

2 Chair of Genomic Medicine, Continental University Medical School, Huancayo, Peru

*Corresponding author: Ramón Cacabelos

rcacabelos@euroespes.com

Institute of Medical Science and Genomic Medicine, EuroEspes Biomedical Research Center, Corunna, Spain.

Citation: Cacabelos R (2017) Some News on Old Concepts and the Epigenetics of Alzheimer's Disease. J Clin Epigenet. Vol.3 No.4:45

with a better understanding of $A D$ pathogenesis in which genomic, epigenomic, metabolic, toxic, and environmental factors converge in a common pathogenic cascade of deleterious events (neuroinflammation, oxidative stress, neurotrophic deficit, proteasome dysfunction, neurotoxicity) leading to progressive neuronal death, and after repetitive failures in different clinical trials with secretase inhibitors/modulators, and especially with vaccines against $A \beta$ deposition (active and passive immunization), the amyloid hypothesis has been challenged from many directions as a reductionist view of $A D[11,12]$ which may still have some future [13]. In the international literature there is also a revival of the vascular component of $A D$ and the importance of vascular dementia as the most prevalent form of dementia in patients over 75 years of age. At this moment, the major challenges posed by $A D$ to the scientific community are the characterization of reliable biomarkers for the preclinical identification of the population at risk in order to implement preventive programs, and the discovery of effective drugs to halt disease progression at pre-symptomatic stages, assuming that the neurodegenerative process leading to AD starts 20-30 years before the onset of the disease [14].

In evolutionary terms, epigenetics is probably a brilliant interface that Nature inserted between the genome and the environmental milieu. In this context, epigenetics is a fundamental process for development, health and disease. Since AD is a disorder of 
the most highly evolved species, it appears obvious that in the crossover of environmental risk factors and genomic defects causing $A D$, epigenetics may have a preponderant role. During the past decade over 500 studies have documented the potential involvement of epigenetics in AD pathogenesis [15]. Although the field of epigenetics in AD is still in its infancy, it seems very likely that alterations in the epigenetic machinery (DNA methylation, histone/chromatin modifications, miRNA regulation) may participate (as primary factors or as secondary events) in the pathogenesis of different neurodegenerative disorders, including $A D$ [15-22]. DNA methylation (5-methylcytosine) (5mC) and DNA hydroxymethylation (5-hydroxymethylcytosine)( $5 \mathrm{hmC})$ are unevenly altered in $A D$ brains. $5 \mathrm{mC}$ is generally associated with the inhibition of gene expression, whereas $5 \mathrm{hmC}$ has been associated with increased gene expression in different processes such as differentiation, development, and aging [23]. Hypermethylation of thousands of $\mathrm{CpG}$ sites have been observed in 485 genes associated with AD in transgenic mice [24]; however, important differences have been found between animal models and humans. Hypo- and hyper-methylated AD genes have been identified in different brain regions, reflecting tissue- and areaspecific epigenetic changes, with conflicting results [16]. Histone modifications have also been reported in $\operatorname{AD}[15,18]$.

Over the past few years, most studies on AD epigenetics have concentrated on ncRNAs. miRNAs are deeply involved in gene expression, influencing diverse pathogenic cascades leading to neurodegeneration [20-22]. The role of miRNAs in the regulation of pathogenic genes associated with AD (APP, BACE1, MAPT, APOE), lipid metabolism, neuroinflammation, and oxidative stress has been extensively documented [18,20-22]. Inducible miRNAs exert regulatory roles in brain development, aging, and neurodegeneration. AD brains show up-regulation of several brain-enriched miRNAs that are under transcriptional control by the pro-inflammatory transcription factor NF-kB, including miRNA-9, miRNA-34a, miRNA-125b, miRNA-146a, and miRNA-155. miRNA-125b is the most abundant and significantly induced miRNA species in human brain cells. Upregulated miRNA-125b may target the 3'-untranslated region (3'-UTR) of the mRNA encoding a 15-lipoxygenase (ALOX15) for the conversion of docosahexaneoic acid into neuroprotectin D1 (NPD1), and the vitamin D3 receptor (VD3R) of the nuclear hormone receptor superfamily. ALOX15 and VD3R are essential factors in lipid-mediated signaling, neurotrophic support, defense against oxidative stress, and neuroprotection. miRNA-125binduced down-regulation of LOX15 and VD3R in the AD brain may alter neurotrophic activity and oxidative stress, contributing to neuronal damage [25]. Some other examples illustrate the role of miRNAs in $A \beta$ formation and deposition [26].

\section{References}

1 GBD 2015 Neurological Disorders Collaborator Group (2017) Global, regional, and national burden of neurological disorders during 19902015: a systematic analysis for the Global Burden of Disease Study 2015. Lancet Neurol 16: 877-897.

2 Chan KY, Wang W, Wu JJ (2013) Global Health Epidemiology
Several signatures of miRNAs have also been proposed as potential biomarkers for $A D$ in peripheral blood [27] and/or in the cerebrospinal fluid [28].

Epigenetic intervention and epigenetic drugs may also come to rescue $A D$ treatment from the misery in which it has remained stagnant for decades, although technical difficulties may preclude a rapid implantation of these procedures [29-33]. Pharmacoepigenetics is becoming a very attractive field, with high complexity $[29,30]$. The genes involved in the pharmacogenetic outcome of $A D$ therapeutics include (i) pathogenic genes associated with $A D$ as potential causative factors, (ii) mechanistic genes whose products participate in the mechanism of action of drugs, (iii) metabolic genes encoding Phase I and Phase II metabolic enzymes, (iv) genes encoding protein transporters, and (v) a vast array of pleiotropic genes involved in multiple metabolomic processes [14,34]. All these genes are under the regulatory control of epigenetic mechanisms, contributing to drug efficacy and safety [35,36].

Some genes may also play dual or multiple roles in the pathogenesis, diagnosis and pharmacoepigenetics of $A D$, such as several members of the ATP Binding Cassette Subfamily (ABCB1, $A B C A 2, A B C A 7)$. For instance, the $A B C A 2$ gene is linked to $A D$ risk and $A B C A 2$ mRNA expression is upregulated in $A D$. Methylation of 2 of 36 CpG islands in the ABCA2 gene with high diagnostic accuracy of $A D$ were found to be negatively associated with $A D$ risk [37]. This pleiotropic gene has also been proposed as a therapeutic target [38].

Some epigenetic drugs, alone or in combination, have demonstrated anti-amyloidotic and neuroprotective effects in $A D$ [39]; however, conventional epigenetic drugs do not easily cross the blood-brain barrier, and their incapability of optimal brain penetration make them a distant therapeutic option in $A D$ while nanoparticle technology is still unable to provide help in brain tissue diffusion. Additionally, gene expression regulation of transporter genes and abnormalities in epigenetic mechanisms regulating metabolic genes may also be responsible for drug resistance in cancer and brain disorders (e.g., epilepsy, depression) $[40,41]$ in different ethnic contexts [42].

As in many other novel fields, much expectation is usually created around epigenetics and pharmacoepigenetics, in part to mitigate the frustration of repetitive failures in the obtaining of reliable biomarkers and effective drugs for $A D$. It is likely that part of the problem is due to the misconception of the animal models currently used in $A D$ research. It would be wise not to replicate the same errors of the past so as not to devalue the potentiality of epigenetics in the future of $A D$ management.

Reference Group (GHERG). Epidemiology of Alzheimer's disease and other forms of dementia in China, 1990-2010: a systematic review and analysis. Lancet 381: 2016-2023.

3 Fiest KM, Roberts JI, Maxwell CJ (2016) The Prevalence and Incidence of Dementia Due to Alzheimer's Disease: a Systematic Review and Meta-Analysis. Can J Neurol Sci 43: S51-82. 
4 Gillespie P, O'Shea E, Cullinan J (2015) Longitudinal costs of caring for people with Alzheimer's disease. Int Psychogeriatr 27: 847-56.

5 Cacabelos R, Teijido O, Carril JC (2016) Can cloud-based tools accelerate Alzheimer's disease drug discovery? Expert Opin Drug Discov 11: 215-223.

6 Selkoe DJ, Hardy J (2016) The amyloid hypothesis of Alzheimer's disease at 25 years. EMBO Mol Med 8: 595-608.

7 Hardy J (2017) The discovery of Alzheimer-causing mutations in the APP gene and the formulation of the "amyloid cascade hypothesis". FEBS J. 284: 1040-1044.

8 Guerreiro R, Hardy J (2014) Genetics of Alzheimer's disease. Neurotherapeutic 11: 732-737.

9 Shao W, Peng D, Wang X (2017) Genetics of Alzheimer's disease: From pathogenesis to clinical usage. J Clin Neurosci 45: 1-8.

10 Cacabelos R, Goldgaber D, Roses AD (2015) Gene interactions in the Pharmacogenomics of Alzheimer's Disease. Sciforschen Genetics and Gene Therapy 1: 1.

11 Kepp KP (2017) Ten Challenges of the Amyloid Hypothesis of Alzheimer's Disease. J Alzheimers Dis 55: 447-457.

12 Ricciarelli R, Fedele E (2017) The Amyloid Cascade Hypothesis in Alzheimer's Disease: It's Time to Change Our Mind. Curr Neuropharmacol 15: 926-935.

13 Karran E, De Strooper B (2016) The amyloid cascade hypothesis: are we poised for success or failure? J Neurochem 2: 237-252.

14 Cacabelos R, Cacabelos P, Torrellas C, Tellado I, Carril JC (2014) Pharmacogenomics of Alzheimer's disease: novel therapeutic strategies for drug development. Methods Mol Biol 1175: 323-556.

15 Cacabelos R, Torrellas C, López-Muñoz F (2014) Epigenomics of Alzheimer's disease. J Exp Med. 6: 75-82

16 Smith RG, Lunnon K (2017) DNA modifications and Alzheimer's disease. In: Delgado-Morales R (Ed). Neuroepigenomics in Aging and Disease. Adv Exp Med Biol 978: 303-319.

17 Narayan P, Dragunow M (2017) Alzheimer's Disease and histone code. In: Delgado-Morales R (Ed). Neuroepigenomics in Aging and Disease. Adv Exp Med Biol.

18 Maoz R, Garfinkel BP, Soreq H (2017) Alzheimer's disease and ncRNAs. In: Delgado-Morales R (Ed). Neuroepigenomics in Aging and Disease. Adv Exp Med Biol 978: 337-361

19 Lu H, Liu X, Deng Y, Qing H (2013) DNA methylation, a hand behind neurodegenerative diseases. Front Aging Neurosci 5: 85.

20 Maciotta S, Meregalli M, Torrente Y (2013) The involvement of microRNAs in neurodegenerative diseases. Front Cell Neurosci 7: 265.

21 Quinlan S, Kenny A, Medina M, Engel T, Jimenez-Mateos EM (2017) MicroRNAs in Neurodegenerative Diseases. Int Rev Cell Mol Biol 334 309-343.

22 Reddy PH, Williams J, Smith F (2017) MicroRNAs, Aging, Cellular Senescence, and Alzheimer's Disease. Prog Mol Biol Transl Sci 146: 127-171.

23 Coppieters N, Dieriks BV, Lill C, Faull RL, Curtis MA, et al. (2014) Global changes in DNA methylation and hydroxymethylation in Alzheimer's disease human brain. Neurobiol Aging 35: 1334-1344.

24 Cong L, Jia J, Qin W, Ren Y, Sun Y (2014) Genome-wide analysis of DNA methylation in an APP/PS1 mouse model of Alzheimer's disease. Acta Neurol Belg 11: 195-206.
25 Zhao Y, Bhattacharjee S, Jones BM, Hill J, Dua P, et al. (2014) Regulation of neurotropic signaling by the inducible, NF-kB-sensitive miRNA-125b in Alzheimer's disease (AD) and in primary human neuronal-glial (HNG) cells. Mol Neurobiol 50: 97-106.

26 Liu H, Chu W, Gong L, Gao X, Wang W (2016) MicroRNA-26b is upregulated in a double transgenic mouse model of Alzheimer's disease and promotes the expression of amyloid- $\beta$ by targeting insulin-like growth factor 1. Mol Med Rep 13: 2809-2814.

27 Rani A, O'Shea A, lanov L, Cohen RA, Woods AJ, et al. (2017) miRNA in Circulating Microvesicles as Biomarkers for Age-Related Cognitive Decline. Front Aging Neurosci 9: 323.

28 Denk J, Boelmans K, Siegismund C, Lassner D, Arlt S, et al. (2015) MicroRNA Profiling of CSF Reveals Potential Biomarkers to Detect Alzheimer's Disease. PLoS One 10: e0126423.

29 Cacabelos R, Torrellas C (2016) Pharmacoepigenomics. In: Medical Epigenetics. T. Tollefsbol (Ed). Elsevier.

30 Cacabelos R, Carril JC, Teijido O (2017) Pharmacogenomics and Epigenomics of Age-Related Neurodegenerative Disorders: Strategies for Drug Development. In: Vaiserman AM (Ed). Anti-aging Drugs: From Basic Research to Clinical Practice; RSC Drug Discovery Series 57: 75-141

31 Cacabelos R, Torrellas C (2015) Epigenetics of aging and Alzheimer's disease: Implications for pharmacogenomics and drug response. Intern J Mol Sci 16: 30483-30543.

32 Cacabelos R, Torrellas C (2014) Epigenetic drug discovery for Alzheimer's disease. Expert Opin Drug Discov 9: 1059-1086.

33 Cacabelos R (2014) Epigenomic networking in drug development: from pathogenic mechanisms to pharmacogenomics. Drug Dev Res 75: 348-365.

34 Cacabelos R, Torrellas C, Carrera I (2015) Opportunities in Pharmacogenomics for the treatment of Alzheimer's Disease. Future Neurology 10: 229-252.

35 Yu A-M, Tian Y, Tu M-J, Ho PY, Jilek JL (2016) MicroRNA Pharmacoepigenetics: Posttranscriptional Regulation Mechanisms behind Variable Drug Disposition and Strategy to Develop More Effective Therapy. Drug Metab Dispos 44: 308-319.

36 Koturbash I, Tolleson WH, Guo L, Yu D, Chen S, et al. (2015) microRNAs as pharmacogenomic biomarkers for drug efficacy and drug safety assessment. Biomark Med 9: 1153-1176.

37 Hu W, Lin X, Zhang H, Zhao N (2017) ATP Binding Cassette Subfamily A Member 2 (ABCA2) Expression and Methylation are Associated with Alzheimer's Disease. Med Sci Monit 23: 5851-5861.

38 Davis W Jr, Tew KD (2017) ATP-binding cassette transporter-2 (ABCA2) as a therapeutic target. Biochem Pharmacol. pii: S0006-2952.

39 Meng J, Li Y, Camarillo C, Yao Y, Zhang Y, et al. (2014) The antitumor histone deacetylase inhibitor SAHA and the natural flavonoid curcumin exhibit synergistic neuroprotection against amyloid-beta toxicity. PLoS One. 9: e85570.

40 Cacabelos R (2015) Pharmacoepigenomics and the metabolomics of drug efficacy and safety. Metabolomics 5: e140.

41 Cacabelos R (2015) Metabolomics of drug resistance in cancer: The Epigenetic Component. Metabolomics 5: e141.

42 Chu S-K, Yang H-C (2017) Interethnic DNA methylation difference and its implications in pharmacoepigenetics. Epigenomics 9: 11. 